\title{
The Impact of the Global and Local Awareness Diffusion on Epidemic Transmission Considering the Heterogeneity of Individual Influences
}

Haidong Xu

Jiangsu University

Ye Zhao

Jiangsu University

Dun Han ( $\square$ handunsir@163.com )

Jiangsu University https://orcid.org/0000-0003-2036-3784

\section{Research Article}

Keywords: Heterogeneity of individual influences, Epidemic transmission, Awareness diffusion, Microscopic Markov chain approach

Posted Date: January 5th, 2022

DOI: https://doi.org/10.21203/rs.3.rs-1080182/v1

License: (9) This work is licensed under a Creative Commons Attribution 4.0 International License. Read Full License 


\title{
The impact of the global and local awareness diffusion on epidemic transmission considering the heterogeneity of individual influences
}

\author{
Haidong Xu · Ye Zhao · Dun Han
}

the date of receipt and acceptance should be inserted later

\begin{abstract}
In this paper, we propose a coupled awareness epidemic spreading model considering the heterogeneity of individual influences, which aims to explore the interaction between awareness diffusion and epidemic transmission. The considered heterogeneity of individual influences are threefold: the heterogeneity of individual influences in the information layer, the heterogeneity of individual influences in the epidemic layer and the heterogeneity of individual behavioral responses to epidemics. In addition, the individuals' receptive preference for information and the impacts of individuals' perceived local awareness ratio and individuals' perceived epidemic severity on self-protective behavior are included. The epidemic threshold is theoretically established according to the microscopic Markov chain approach and mean-field approach. Results indicate that the critical local and global awareness ratios have two-stage effects on the epidemic threshold. Besides, either the heterogeneity of individual influences in the information layer or the strength of individuals' responses to epidemics can influence the epidemic threshold with a nonlinear way. However, the heterogeneity of individual influences in the epidemic layer has few effect on the epidemic threshold, but can affects the magnitude of the final infected density.
\end{abstract}

Keywords Heterogeneity of individual influences · Epidemic transmission - Awareness diffusion - Microscopic Markov chain approach

H. Xu $\cdot$ Y. Zhao $\cdot$ D. Han $(\bowtie)$

School of Mathematical Sciences, Jiangsu University, Zhenjiang, Jiangsu 212013, China

E-mail: handunsir@163.com

\section{Introduction}

Throughout history, the outbreak of epidemics has been a major disaster threatening human survival. For example, from 2019 to now, the sudden outbreak of COVID-19 [1-4] has deeply affected the lifestyle of people all over the world and has an indelible negative impact on the world economy. During the outbreak of COVID-19, epidemiological information is transmitted by word of mouth or social media, etc [5-9]. To avoid being infected by a disease, human behaviors may change such as reducing outdoor activities, wearing masks, paying attention to disinfection and vaccination, etc [10-13]. Therefore, more and more researchers focus on the interaction between awareness diffusion and epidemic transmission [14-17]. Funk et al. proposed a mathematical to model the interplay between epidemic and awareness [18]. Wang et al. established an asymmetrically coupled epidemic-awareness spreading model, and found that the information threshold remained unchanged and the epidemic threshold could be increased if structural correlation existed between the contact layer and the communication layer [19].

Due to the development of technology and society, people can convenient obtain epidemiological information through different ways. Yet, people access to information mainly from their acquaintance and the news media, that are classed as local information [20,21,27] and the global information [14, $24]$ respectively. On the other hand, people usually take different behaviors according to the information they received. Consequently, a large number of scholars have analyzed how the diffusion of information affects the spread of infectious diseases. Some researchers considered that individuals could be directly or indirectly affected by different awareness in the process of epidemic transmission. For instance, Guo et al. investigated the effects of local awareness on the coupled UAU-SIS model. The result indicated that the local awareness ratio has profound effects on epidemic threshold [22]. 
Kan et al. assumed that the susceptible individuals could be self-awareness affected by their infected neighbors, and they proved that self-awareness could reduce the infected density but could not enhance the epidemic threshold [23]. Zuo et al. found that the epidemic spreading could be affected by the effective transmission rate of the awareness and neighbors' behavior status [24]. Other researchers had studied the influence of various factors in the awareness transmission on epidemic spreading from a macroscopic perspective. For example, Wang et al. studied the co-evolution spreading of positive information, negative information and epidemic under the influence of mass media. They found that enhancing information dissemination and the propaganda of media could suppress the epidemic spreading [25]. Zhang et al. researched the impacts of awareness diffusion, individual vigilance and rational behavior on epidemic transmission. Their result indicated that the rational behavior played an important role in controlling epidemic transmission [26]. Moreover, many researchers had investigated the influence of awareness transmission on epidemic spreading considering the heterogeneity of individuals. Pan et al. explored the impacts of the heterogeneity of influences in the information, the heterogeneity of influences in the epidemic layer and the heterogeneity of individual responses to epidemics on epidemic spreading [27]. Scata et al. utilized real data to examine the joint impacts of heterogeneity and awareness on epidemic transmission in different socioeconomic conditions [28]. Chen et al. investigated the effects of heterogeneous self-awareness distribution on the epidemic dynamic. The result showed that the heterogeneity of self-awareness distribution could suppress the outbreak of an disease [29].

Most of previous studies related to epidemic spreading mainly focus on network structure, information diffusion and individual behavioral responses. However, almost all of studies assumed that all individuals were treated equally [21]. In other words, the infection or awareness rate was the same for each individual. Obviously, it is not consistent with the real situation. In fact, different people has different sensitivity or reactions to infectious diseases and information. Therefore, the heterogeneity of individuals should be considered while modeling the dynamic processes between epidemic transmission and information diffusion.

In this paper, to explore the impact of the global and local awareness diffusion on epidemic transmission, we propose a coupled awareness-epidemic spreading model by considering the heterogeneity of individual influences in the information layer, the heterogeneity of individual influences in the epidemic layer and the heterogeneity of individual behavioral responses to epidemics. The results show that the heterogeneity of individual influences in the epidemic layer has no effect on the epidemic threshold, but it can affects the magnitude of the final density of infected individuals. In addition, we find that both the heterogeneity of individual influ-

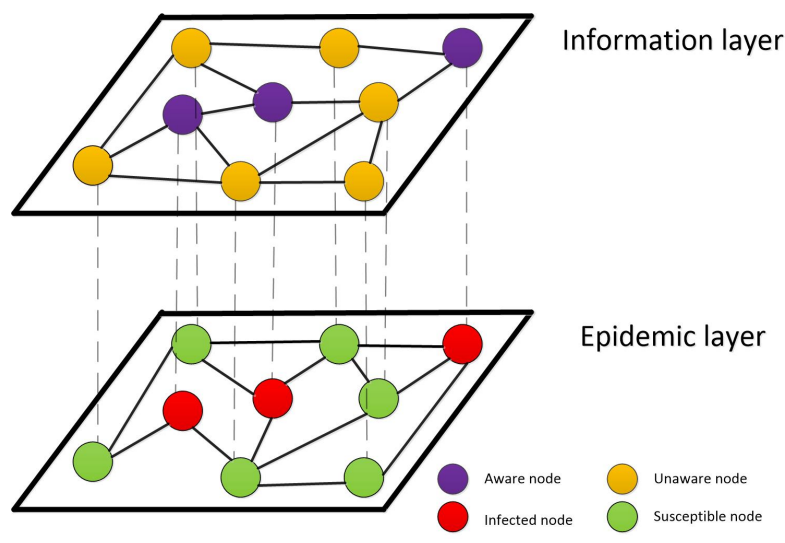

Fig. 1 An coupled awareness - epidemic spreading model in the two layer networks. Epidemic transmission in the epidemic layer, and nodes have two possible states for each node: Susceptible (S) or Infected (I). Awareness diffusion in the information layer, and nodes have two possible states for each node: Aware (A) or Unaware (U).

ences in the information layer and the strength of individuals' responses to epidemics have a nonlinear effect on the size of the epidemic threshold. The contributions of this paper are threefold as follows. (1) Using the threshold model, we present and analyze the epidemic spreading problem with the global awareness diffusion; (2) Individuals' perceived local awareness ratio and individuals' self-protective behavior are incorporated into the proposed epidemic spreading model; (3) We confirm that individuals' information receptive preference has a powerful influence on epidemic spreading.

The rest of this paper is organized as follows: in Sec.2, we introduce a coupled awareness-epidemic spreading model considering the heterogeneity of individual influences. Then, in Sec.3, we use the microscopic Markov chain approach (MMCA) to analyze the model and derive the analytical expression of the epidemic threshold. In Sec.4, according to the simulation results, we explore the impact of the heterogeneity of individual influence on the the interplay of epidemics and awareness. Finally, we conclude the paper in Sec.5.

\section{Model Description}

We employ an SIS (Susceptible-Infected-Susceptible) model to describe the process of epidemic transmission. As shown in Fig. 1, the information layer describes the process of awareness diffusion, where links represent the virtual contacts among individuals. In this layer, there are two possible states for each node: Aware (A) or Unaware (U), which means that individuals are aware or unaware of epidemics, respectively. The epidemic layer describes the process of epidemic transmission, where links represent the physical contacts among individuals. In this layer, the state of each node is the one of two states: Susceptible (S) or Infected (I), which means that individuals are susceptible or infected, respectively. For the 
sake of simplicity, both the information layer network and the epidemic layer network are undirected and unweighted. Furthermore, there is a one to one correspondence between the nodes of these two layer networks, and different layers have different topologies.

In the information layer, an unaware individual becomes aware when its perceived local awareness ratio $A_{i}^{\text {perc }}$ exceeds the critical local awareness ratio $\theta_{1}$. Otherwise, the individual will continue to be unaware. Moreover, all individuals become aware when the average awareness rate of all individuals exceeds the critical global awareness $\theta_{2}$. In addition, an aware individual becomes unaware with probability $\delta$. In the epidemic layer, a susceptible individual gets infected with probability $\beta$ after a contact with an infected individual, and an infected individual recovers to be susceptible with probability $\mu$.

For simplicity, we assume that infected individuals become aware in the information layer. Obviously, susceptible individuals will take self-protective measures to avoid being infected if they become aware in the information layer. Therefore, the probability of individual $i$ being infected is affected by the status of individual $i$ in the information layer. If an susceptible individual $i$ is an unaware individual in the information layer, the individual gets infected with probability $\beta^{U}=\beta$. If an susceptible individual $i$ is an aware individual in the information layer, the individual gets infected with probability $\beta_{i}^{A}=\gamma_{i} \beta^{U}\left(\gamma_{i} \in[0,1]\right)$. The parameter $\gamma_{i}$ regulates the probability of an aware individual $i$ being infected. $\gamma_{i}=0$ denotes that the aware individual $i$ is completely immune to the infection. $\gamma_{i}=1$ denotes that the aware individual $i$ is ineffective to reduce the infectivity of the epidemic by taking protective measures. In previous studies, researchers assumed that aware individuals would take equivalent protective measures and have the same risk of being infected, ignoring the heterogeneous influence of individuals on the epidemic. Then, we extend our proposed model by introducing three types of heterogeneity.

Firstly, we introduce the heterogeneity of individual influences in the information layer. In the information layer, different individuals may have different influences on others. The rate of individuals' perceived local awareness depends on the state and influence of their neighbors. We assume that $A_{i}^{\text {perc }}$ and $w_{i j}$ represents the perceived local awareness ratio of individual $i$ [27] and the information reception rate after mutual sharing of information between individuals $i$ and $j$, respectively. Then, $A_{i}^{\text {perc }}$ can be described as follows:

$A_{i}^{\text {perc }}=\sum_{j \in \Gamma_{i}} w_{i j} \cdot A_{j}$

In Eq.(1), $\Gamma_{i}$ represents neighbors set of individual $i$ in the information layer and $A_{j}$ denotes whether individual $j$ is an awareness individual. If individual $j$ is an awareness individual, $A_{j}=1$. Otherwise, $A_{j}=0$.
Considering that neighbors with different node degrees have different influences on individual $i$, we define $T_{i j}$ as

$T_{i j}=\frac{k_{j}^{\alpha}}{\sum_{s \in \Gamma_{i}} k_{s}^{\alpha}}$

where $T_{i j}$ denotes the influences of individual $j$ on individual $i . k_{j}$ represents the node degree of individual $j$ in the information layer. $\alpha$ controls the heterogeneity of individual influences in the information layer, which $\alpha$ takes an arbitrary constant.

In fact, in the process of information diffusion, each individual's process of transmitting and receiving information are independent of each other, and individuals receive information from different individuals with different preferences. These factors jointly determine how well an individual understands the information. Thus, we define $w_{i j}$ as

$w_{i j}=\pi T_{i j}+(1-\pi) T_{j i}$

where $\pi$ represents the receptive preference of individual $i$ for the information shared by individual $j, 1-\pi$ denotes the receptive preference of individual $j$ for the information shared by individual $i, \pi \in[0,1]$. When $\pi=0, w_{i j}$ is determined only by $T_{j i}$. When $\pi=1, w_{i j}$ is determined only by $T_{i j}$.

Secondly, we introduce the heterogeneity of individual influences in the epidemic layer. Duing to their geographic location and contact environment, individuals have different perceptions of epidemic severity [27] in the epidemic layer. Let $B_{i}^{\text {perc }}$ denotes the perceived epidemic severity of individual $i, w_{i j}^{\prime}$ represents the influence of individual $j$ on individual $i$ in the epidemic layer. $B_{i}^{\text {perc }}$ can be described as follows:

$B_{i}^{\text {perc }}=\sum_{j \in \Gamma_{i}^{\prime}} w_{i j}^{\prime} \cdot B_{j}$

where $\Gamma_{i}^{\prime}$ represents neighbors set of individual $i$ in the epidemic layer and $\boldsymbol{B}_{\boldsymbol{j}}$ denotes whether individual $\boldsymbol{j}$ is an infected individual. If individual $j$ is an infected individual, $B_{j}=1$. Otherwise, $B_{j}=0$.

Considering that individuals with different node degrees have different influences on individual $i$, we define $w_{i j}^{\prime}$ as

$w_{i j}^{\prime}=\frac{k_{j}^{\prime \eta}}{\sum_{s \in \Gamma_{i}^{\prime}} k_{s}^{\prime \eta}}$

In Eq.(5), $k_{j}^{\prime}$ represents the node degree of individual $j$ in the epidemic layer and $\eta$ controls the heterogeneity of individual influences in the epidemic layer, which $\eta$ takes an arbitrary constant.

Finally, we introduce the heterogeneity of individual behavioral responses to epidemics. Apparently, if a susceptible individual $i$ becomes aware in the information layer, they 
will take self-protective measures and reduce their infectivity $\beta$. Let $\beta^{U}$ and $\beta_{i}^{A}$ be the infection probabilities of unaware individuals and aware individuals respectively. In fact, If individuals have a high perceived local awareness ratio and a high perceived epidemic severity, individuals will become more cautious and take more effective protective measures. We assume that self-protective measures taken by individuals are mainly determined by their perceived local awareness ratio and their perceived epidemic severity. Thus, $\beta_{i}^{A}$ can be described as

$\beta_{i}^{A}=\gamma_{i} \beta=\beta(1-\lambda)^{k_{i} \cdot\left(\tau A_{i}^{\text {perc }}+(1-\tau) B_{i}^{\text {perc }}\right)}$

where $\tau$ denotes the weight of individuals' perceived local awareness ratio, and $1-\tau$ represents the weight of individuals' perceived epidemic severity, $\tau \in[0,1]$. When $\tau=0, \beta_{i}^{A}$ is determined only by individuals' perceived epidemic severity. When $\tau=1, \beta_{i}^{A}$ is determined only by individuals' perceived local awareness ratio. The parameter $\lambda$ represents the strength of an individuals' responses to epidemics. In particular, $\gamma_{i}$ equals 0 for $\lambda=1$, representing total immunization, and $\gamma_{i}$ equals 1 for $\lambda=0$, representing self-protective measures taken are not effective for epidemics.

\section{Analytical Results Based On MMCA}

According to our proposed model, the $N$ nodes in the multiplex model can be in the following states: unawareness susceptible (US), awareness - susceptible (AS), awareness - infected (AI). For ease of analysis, we assume that both awareness diffusion and epidemic transmission are Markovian processes $[7,9,11,14,15,17,18,22,24-27]$. We assume that the probabilities that individual $i$ being in the above three states at time $t$ as $p_{i}^{U S}(t), p_{i}^{A S}(t), p_{i}^{A I}(t)$. Evidently, each time step is satisfied $p_{i}^{U S}(t)+p_{i}^{A S}(t)+p_{i}^{A I}(t)=1$.

Let $A=\left(a_{i j}\right)_{N \times N}$ and $B=\left(b_{i j}\right)_{N \times N}$ be the adjacency matrices of the information layer network and the epidemic layer network, respectively. Furthermore, in the information layer, the probability that individual $i$ not being informed of epidemiological information by any neighbors is defined as $r_{i}(t)$, and the probability that individual $i$ not being informed of epidemiological information by mass media is defined as $m_{i}(t)$. In the epidemic layer, the probabilities that an aware or unaware susceptible individual has not been infected are defined as $q_{i}^{A}(t)$ or $q_{i}^{U}(t)$. Thus, $r_{i}(t), m_{i}(t), q_{i}^{A}(t)$ and $q_{i}^{U}(t)$ can be described as follows:
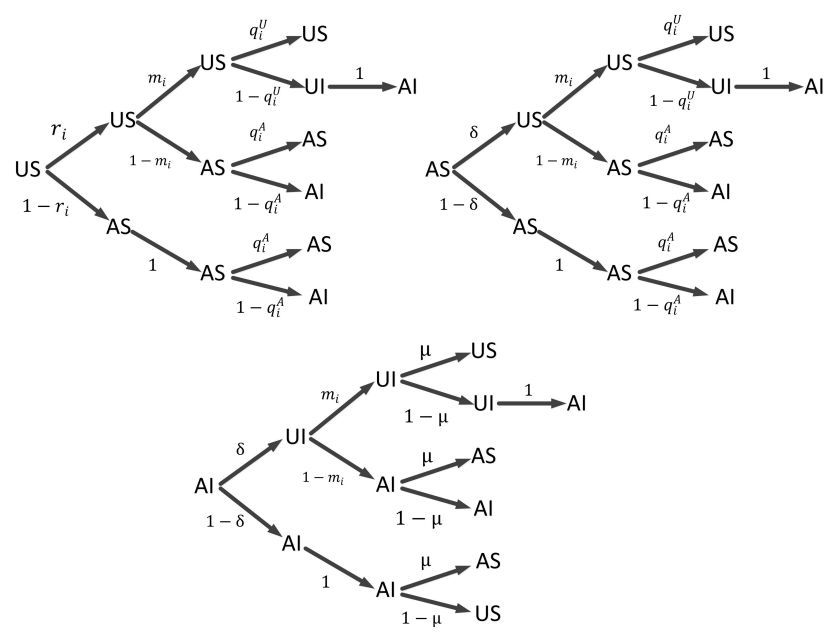

Fig. 2 Transition probability tree of the three states in the model. $r_{i}(t)$ and $m_{i}(t)$ denotes the probability that individuals $i$ not being informed of the epidemiological information by any neighbors or mass media, respectively. $\delta$ represents the probability that awareness individuals forget the epidemiological information. $q_{i}^{A}(t)$ and $q_{i}^{U}(t)$ denotes the probabilities that an aware and unaware susceptible individual has not been infected, respectively. $\mu$ represents the probability that an infected individual recovers to be susceptible.

$$
\left\{\begin{array}{l}
r_{i}(t)=H\left[\theta_{1}-A_{i}^{\text {perc }}\right] \\
m_{i}(t)=H\left[\theta_{2}-\frac{\sum_{j=1}^{N} p_{j}^{A}(t)}{N}\right] \\
q_{i}^{A}(t)=\prod_{j}\left[1-b_{j i} p_{j}^{A I} \gamma_{i} \beta\right] \\
q_{i}^{U}(t)=\prod_{j}\left[1-b_{j i} p_{j}^{A I} \beta\right]
\end{array}\right.
$$

where $\theta_{1}$ and $\theta_{2}$ denotes the critical local awareness ratio and the critical global awareness ratio, respectively. $H(x)$ is a Heaviside step function, $H(x)$ equals 1 for $x>0$, which represents the unaware individual will continue to be unaware; $H(x)$ equals 0 for $x \leqslant 0$, which represents the unaware individual will be aware by their neighbors or mass media.

Then, we construct a transition probability tree, as shown in Fig.2. In addition, the perceived local awareness of individual $i$ and the perceived epidemic severity of individual $i$ are defined as $A_{i}^{\text {perc }}=\sum_{j} a_{i j} w_{i j} p_{j}^{A}(t)$ and $B_{i}^{\text {perc }}=\sum_{j} b_{i j} w^{\prime}{ }_{i j}$ $p_{j}^{A}(t)$, respectively. where $p_{j}^{A}(t)=p_{j}^{A S}(t)+p_{j}^{A I}(t)$.

According to the microscopic Markov chain approach (MMCA), we obtain the following equation of dynamic evo- 
lution:

$$
\left\{\begin{aligned}
p_{i}^{U S}(t+1) & =p_{i}^{U S}(t) r_{i}(t) m_{i}(t) q_{i}^{U}(t) \\
& +p_{i}^{A S}(t) \delta m_{i}(t) q_{i}^{U}(t)+p_{i}^{A I}(t) \delta m_{i}(t) \mu \\
p_{i}^{A S}(t+1) & =p_{i}^{U S}(t)\left\{r_{i}(t)\left[1-m_{i}(t)\right]+\left[1-r_{i}(t)\right]\right\} q_{i}^{A}(t) \\
& +p_{i}^{A S}(t)\left\{\delta\left[1-m_{i}(t)\right]+(1-\delta)\right\} q_{i}^{A}(t) \\
& +p_{i}^{A I}(t)\left\{\delta\left[1-m_{i}(t)\right]+(1-\delta)\right\} \mu \\
p_{i}^{A I}(t+1) & =p_{i}^{U S}(t) r_{i}(t) m_{i}(t)\left[1-q_{i}^{U}(t)\right] \\
& +p_{i}^{U S}(t)\left\{r_{i}(t)\left[1-m_{i}(t)\right]+\left[1-r_{i}(t)\right]\right\}\left[1-q_{i}^{A}(t)\right] \\
& +p_{i}^{A S}(t) \delta m_{i}(t)\left[1-q_{i}^{U}(t)\right] \\
& +p_{i}^{A S}(t)\left\{\delta\left[1-m_{i}(t)\right]+(1-\delta)\right\}\left[1-q_{i}^{A}(t)\right] \\
& +p_{i}^{A I}(t)(1-\mu)
\end{aligned}\right.
$$

When the time step is large enough, the proportions of states for each node reach a steady state. Thus, when $t \rightarrow+\infty$, we obtain:

$$
\left\{\begin{array}{l}
p_{i}^{U S}(t+1)_{t \rightarrow+\infty}=p_{i}^{U S}(t)_{t \rightarrow+\infty}=p_{i}^{U S} \\
p_{i}^{A S}(t+1)_{t \rightarrow+\infty}=p_{i}^{A S}(t)_{t \rightarrow+\infty}=p_{i}^{A S} \\
p_{i}^{A I}(t+1)_{t \rightarrow+\infty}=p_{i}^{A I}(t)_{t \rightarrow+\infty}=p_{i}^{A I}
\end{array}\right.
$$

Near the onset of epidemics, the probability of nodes to be infected is close to zero. Thus, we assume that $p_{i}^{A I}=\varepsilon_{i} \leqslant$ $1, \varepsilon_{i}>0$, and Eqs.(9) and (10) are approximated as:

$$
\left\{\begin{array}{l}
q_{i}^{A}=\prod_{j}\left[1-b_{i j} p_{i}^{A I}(t) \gamma_{i} \beta\right] \approx 1-\gamma_{i} \beta \sum_{j} b_{i j} \varepsilon_{j} \\
q_{i}^{U}=\prod_{j}\left[1-b_{i j} p_{i}^{A I}(t) \beta\right] \approx 1-\beta \sum_{j} b_{i j} \varepsilon_{j}
\end{array}\right.
$$

where

$$
\left\{\begin{array}{c}
\sigma_{i}^{A}=\gamma_{i} \beta \sum_{j} b_{j i} \varepsilon_{j} \\
\sigma_{i}^{U}=\beta \sum_{j} b_{j i} \varepsilon_{j}
\end{array}\right.
$$

Then, $q_{i}^{A}$ and $q_{i}^{U}$ can be described as $q_{i}^{A} \approx 1-\sigma_{i}^{A}$ and $q_{i}^{U} \approx 1-\sigma_{i}^{U}$, respectively. We substitute Eqs.(15) and (16) into Eq.(11), and ignore the higher-order terms $O\left(\varepsilon_{i}\right)$ to obtain:

$$
\left\{\begin{aligned}
p_{i}^{U S} & =p_{i}^{U S} r_{i} m_{i}+p_{i}^{A S} \delta m_{i} \\
p_{i}^{A S} & =p_{i}^{U S}\left(1-r_{i} m_{i}\right)+p_{i}^{A S}\left(1-\delta m_{i}\right) \\
\mu \varepsilon_{i} & =p_{i}^{U S}\left[r_{i} m_{i} \sigma_{i}^{U}+\left(1-r_{i} m_{i}\right) \sigma_{i}^{A}\right] \\
& +p_{i}^{A S}\left[\delta m_{i} \sigma_{i}^{U}+\left(1-\delta m_{i}\right) \sigma_{i}^{A}\right]
\end{aligned}\right.
$$

Because $p_{i}^{A I} \rightarrow 0$ and $p_{i}^{A}=p_{i}^{A S}+p_{i}^{A I}, p_{i}^{A} \approx p_{I}^{A S}$ and $1-$ $p_{i}^{A} \approx p_{i}^{U S}$. Inserting Eqs.(17) and (18) in Eq.(21), we obtain

$$
\begin{aligned}
\mu \varepsilon_{i} & =p_{i}^{U S}\left[r_{i} \sigma_{i}^{U}+\left(1-r_{i}\right) \sigma_{i}^{A}\right]+p_{i}^{A S}\left[(1-\delta) \sigma_{i}^{A}+\delta \sigma_{i}^{U}\right] \\
& =p_{i}^{U S} \sigma_{i}^{U}+p_{i}^{A S} \gamma_{i} \sigma_{i}^{A} \\
& =\left(1-p_{i}^{A S}\right) \sigma_{i}^{U}+p_{i}^{A S} \gamma_{i} \sigma_{i}^{U} \\
& =\left[1-\left(1-\gamma_{i}\right) p_{i}^{A}\right] \beta \sum_{j} b_{j i} \varepsilon_{j}
\end{aligned}
$$

In addition, Eq.(22) can be rewritten as

$$
\sum_{j}\left\{\left[1-\left(1-\gamma_{i}\right) p_{i}^{A}\right] b_{j i}-\frac{\mu}{\beta} \zeta_{i j}\right\} \varepsilon_{j}=0
$$

where $\zeta_{i j}$ is the element of the identity matrix. Let $\Phi_{i j}=$ $\left[1-\left(1-\gamma_{i}\right) p_{i}^{A}\right] b_{j i}$ be the element of matrix $\Phi$. Then, Eq.(23) can be rewritten as

$\Phi E=\frac{\mu}{\beta} E$

where $E=\left(\epsilon_{1}, \epsilon_{2}, \ldots, \epsilon_{N}\right)^{T}$. Defining the nontrivial solutions of Eq.(24) are eigenvectors of $\Phi$, whose largest real eigenvalues are equal to $\frac{\mu}{\beta}$. Therefore, the epidemic threshold $\beta_{c}$ is to satisfy $\frac{\mu}{\beta}=\Lambda_{\max }(\Phi)$, where $\Lambda_{\max }(\Phi)$ denotes that the maximum eigenvalue of the matrix $\Phi$. Then, the epidemic threshold can be described as

$\beta_{c}=\frac{\mu}{\Lambda_{\max }(\Phi)}$

Based on Eqs.(24) and (25), the epidemic threshold $\beta_{c}$ depends on the infection attenuation factor $\gamma_{i}$ and the density of aware individuals in the information layer $p_{i}^{A}$, where $p_{i}^{A}$ is further determined by the network structure, the recovery rate $\mu$ and the forgetting rate $\delta$.

\section{Numerical Simulation}

According to Eq.(11), given the initial conditions, we can obtain the probabilities of each individual being in a different state at any time by iterations. The stationary fraction of aware individuals and infected individuals can be described as $\rho^{A}=\sum_{i}\left(p_{i}^{A S}+p_{i}^{A I}\right) / N$ and $\rho^{I}=\sum_{i} p_{i}^{A I} / N$, respectively.

Considering the heterogeneity of individual influences on others is based on the degree of nodes, we construct a two-layer BA scale-free network [30] with no correlation between layers. There are totally $N=1000$ nodes in each layer network, and the average degree of BA networks is set as $\langle k\rangle=5.98$. The initial infection fraction $I_{0}=0.1$.

Firstly, we use Monte Carlo (MC) simulations to test the accuracy of MMCA. As shown in Fig.3, the results of MMCA 


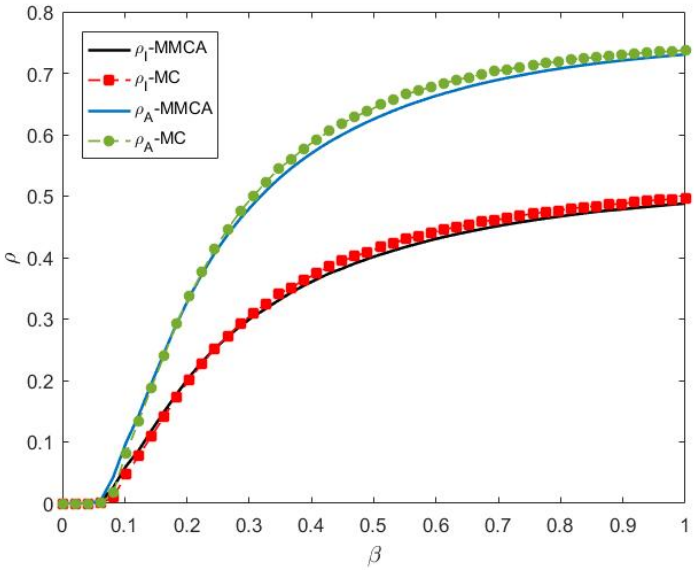

Fig. 3 Comparison of $\rho^{I}$ and $\rho^{A}$ as a function of $\beta$ using MMCA and Monte Carlo (MC) simulations. The black solid line and blue solid line are $\rho^{I}$ and $\rho^{A}$ using MMCA, The red squares and green dots are $\rho^{I}$ and $\rho^{A}$ using MC simulations, respectively. All simulation results are averaged by 300 realizations. The remaining parameters are $\theta_{1}=0.9$, $\theta_{2}=0.9, \mu=0.8, \delta=0.6, \alpha=2, \eta=2, \lambda=0.5, \tau=0.2, \pi=0.5$.
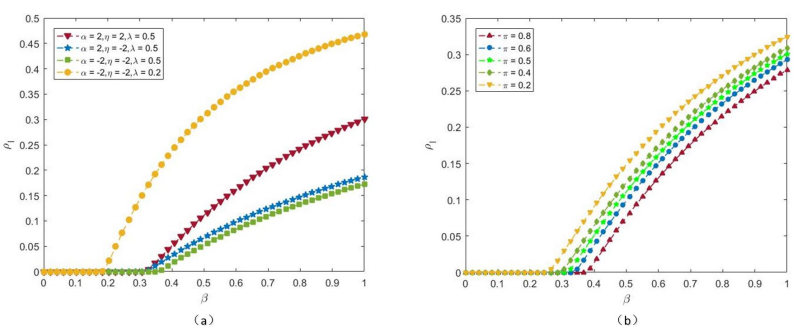

Fig. 4 (a) $\rho^{I}$ as a function of $\beta$ for different values of $\alpha, \eta$ and $\lambda$, where $\pi=0.5$. (b) $\rho^{I}$ as a function of $\beta$ for different values of $\pi$, where $\alpha=2$, $\eta=2, \lambda=0.5$. The remaining parameters are $\theta_{1}=0.1, \theta_{2}=0.1, \mu=0.8$, $\delta=0.6, \tau=0.2$.

and MC simulations for $\rho^{I}$ and $\rho^{A}$ are in agreement, which demonstrates the accuracy of MMCA in solving the coupling dynamics proposed in this paper. Then, we use MMCA to analyze the relationship between $\beta$ and $\rho^{I}$ at different types of heterogeneity and individuals' receptive preferences for information, respectively.

In Fig.4, we mainly consider the relationship between $\beta$ and $\rho^{I}$ when the individuals are all informed about the epidemic. As shown in Fig.4 (a), $\rho^{I}$ decreases as $\lambda$ increases for the same sets of $\alpha$ and $\eta$. Therefore, improving the strength of the individuals' responses to epidemics can effectively reduce the final density of infected individuals and suppress the outbreak of an epidemic. In addition, we find that decreasing $\alpha$ and $\eta$ can reduce the final density of infected individuals, and suppress the epidemic transmission.

Noticed that reducing the final density of infected individuals $\rho^{I}$ by the parameter $\eta$ has a greater effect than decreasing $\rho^{I}$ by the parameter $\alpha$. In reality, epidemics can spread only in the physical contact layer, but not in the information dissemination. However, the information transmis-
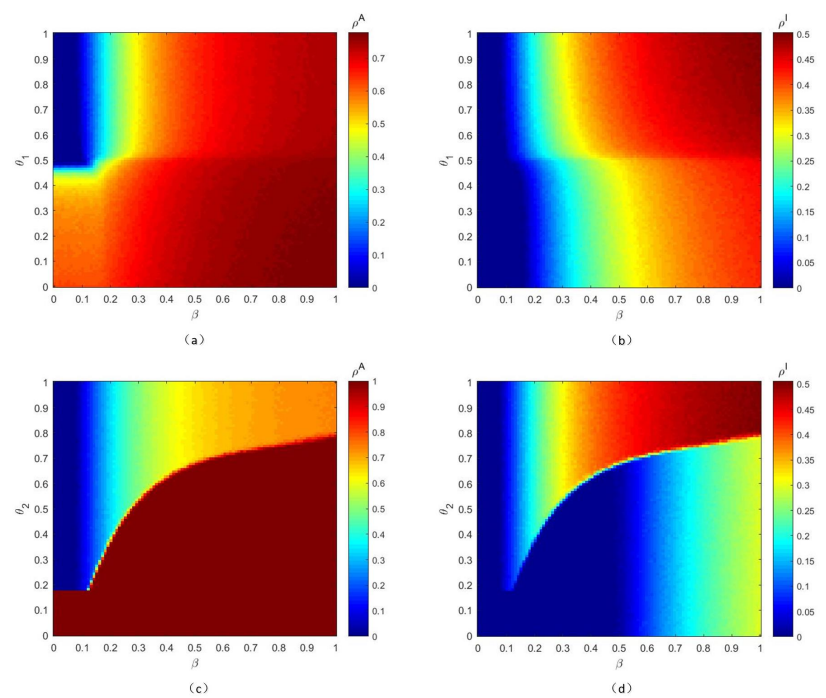

Fig. $5(a)-(b)$ The full phase diagrams $\left(\theta_{1}-\beta\right)$ of $\rho^{A}$ and $\rho^{I}$, respectively, where $\theta_{2}=1$. $(c)-(d)$ The full phase diagrams $\left(\theta_{2}-\beta\right)$ of $\rho^{A}$ and $\rho^{I}$, respectively, where $\theta_{1}=1$. The four phase diagrams are obtained by averaging $50 \mathrm{MC}$ simulations for each point in the grid $100 \times 100$. The remaining parameters are $\mu=0.8, \delta=0.6, \alpha=2, \eta=2, \lambda=0.5, \tau=0.2$, $\pi=0.5$.

sion layer can warn people to take appropriate self protective measures by spreading the epidemiological information. Furthermore, in Fig.4 (b), we observe that $\rho^{I}$ decreases as $\pi$ increases. This indicates that in the process of epidemic transmission, the more individuals can accept information shared by others, the more beneficial to suppress the outbreak of an epidemic.

Our proposed model show that the critical local awareness ratio $\theta_{1}$ and the critical global awareness ratio $\theta_{2}$ controls the threshold of being aware. When only the local or global awareness is regarded, we investigate the impacts of the critical local awareness ratio $\theta_{1}$ and the critical global awareness ratio $\theta_{2}$ on the epidemic transmission and the awareness diffusion, respectively. Thus, we plot the four related phase diagrams, as shown in Fig.5.

In Fig.5, we find the thresholds for $\theta_{1}$ and $\theta_{2}$, respectively. If $\theta_{1}$ or $\theta_{2}$ is less than its threshold, both $\rho^{A}$ and $\rho^{I}$ have an abrupt transition, i.e., $\rho^{A}$ will abruptly increase and $\rho^{I}$ will suddenly decrease. In addition, we also find that the threshold for $\theta_{2}$ increases as the infection rate $\beta$ enhances. The results show that improving the awareness diffusion among individuals and the publicity of epidemic by mass media is an effective way to suppress the epidemic transmission.

Furthermore, we examine the impacts of the critical local and global awareness on the epidemic threshold, respectively. In Fig.6, we find that both $\theta_{1}$ and $\theta_{2}$ have two-stage effects on the epidemic threshold $\beta_{c}$. Apparently, the effect of $\theta_{2}$ on $\beta_{c}$ is larger than that of $\theta_{1}$ on $\beta_{c}$. Besides, as the parameter $\alpha$ reduces, the value of $\theta_{1}$ which make $\beta_{c}$ undergo an abrupt transition decreases. In addition, the value of a low 

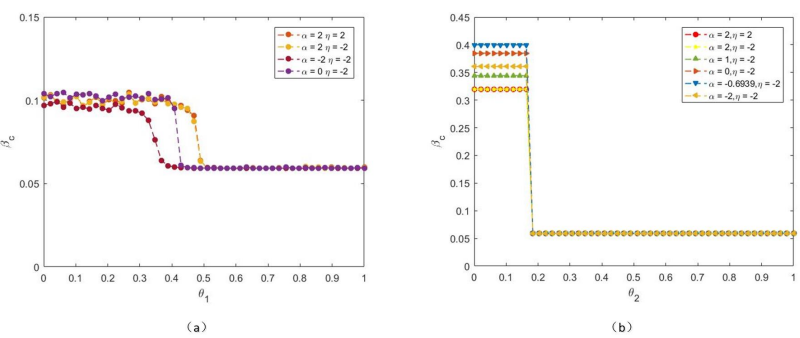

Fig. 6 (a) $\beta_{c}$ as a function of $\theta_{1}$ for different values of $\alpha$ and $\eta$, where $\theta_{2}=1$. (b) $\beta_{c}$ as a function of $\theta_{2}$ for different values of $\alpha$ and $\eta$, where $\theta_{1}=1$. The two diagrams are obtained by averaging $100 \mathrm{MC}$ simulations. The remaining parameters are $\mu=0.8, \delta=0.6, \lambda=0.5, \tau=0.2$, $\pi=0.5$.
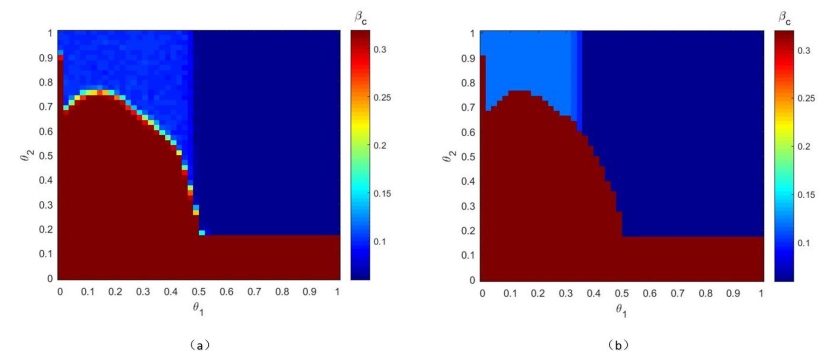

Fig. 7 (a) The full phase diagrams $\left(\theta_{1}-\theta_{2}\right)$ of $\beta_{c}$ derived from MC simulations. This phase diagram is obtained by averaging $50 \mathrm{MC}$ simulations for each point in the grid $50 \times 50$. (b) The full phase diagrams $\left(\theta_{1}-\theta_{2}\right)$ of $\beta_{c}$ derived from MMCA. The remaining parameters are $\mu=0.8, \delta=0.6, \alpha=2, \eta=2, \lambda=0.5, \tau=0.2, \pi=0.5$.

phase of the epidemic threshold (i.e., the smaller $\beta_{c}$ in Fig.6) is independent of $\alpha$ and $\eta$, which is similar with previous studies [27]. However, the value of $\beta_{c}$ for $\theta_{2}$ are different from the value of $\beta_{c}$ for $\theta_{1}$. Compared Fig.6 $(b)$ with Fig.8 (c), the value of a high phase of the epidemic threshold is related to the value of $\beta_{c}$ for $\alpha$ when only $\theta_{2}$ is considered. That is, the larger value of $\beta_{c}$ for $\alpha$, the greater value of the high phase of the epidemic threshold. Noticed that the value of $\beta_{c}$ for $\alpha$ is close to its maximum when $\alpha=-0.6939$.

In Fig.6, we only consider the impact of the critical local awareness $\theta_{1}$ ( or the critical global awareness $\theta_{2}$ ) on the epidemic threshold $\beta_{c}$. In order to explore the impacts of different $\theta_{1}$ and $\theta_{2}$ on the epidemic threshold $\beta_{c}$, we utilize MC simulation and MMCA to derive the full phase diagrams $\left(\theta_{1}-\theta_{2}\right)$ of $\beta_{c}$, respectively.

As shown in Fig.7, we observe a discrepancy between Fig.7 (a) and Fig.7 (b). In the analytical results of MMCA, the threshold of abrupt transition of $\beta_{c}$ for $\theta_{1}$ is smaller than that shown in the results of MC simulations. Note that Eqs.(7)(8) are obtained based on the assumption of an absence of dynamical correlation $[9,14,27,31]$. At this assumption, the states of the nodes among neighbors should be independent [9,27,32]. However, dynamical correlations may exist among the states of neighbors, especially in networks with a high clustering coefficient $[9,27]$. Thus, the discrepancy may exist between MC simulations and MMCA. In addition, we find
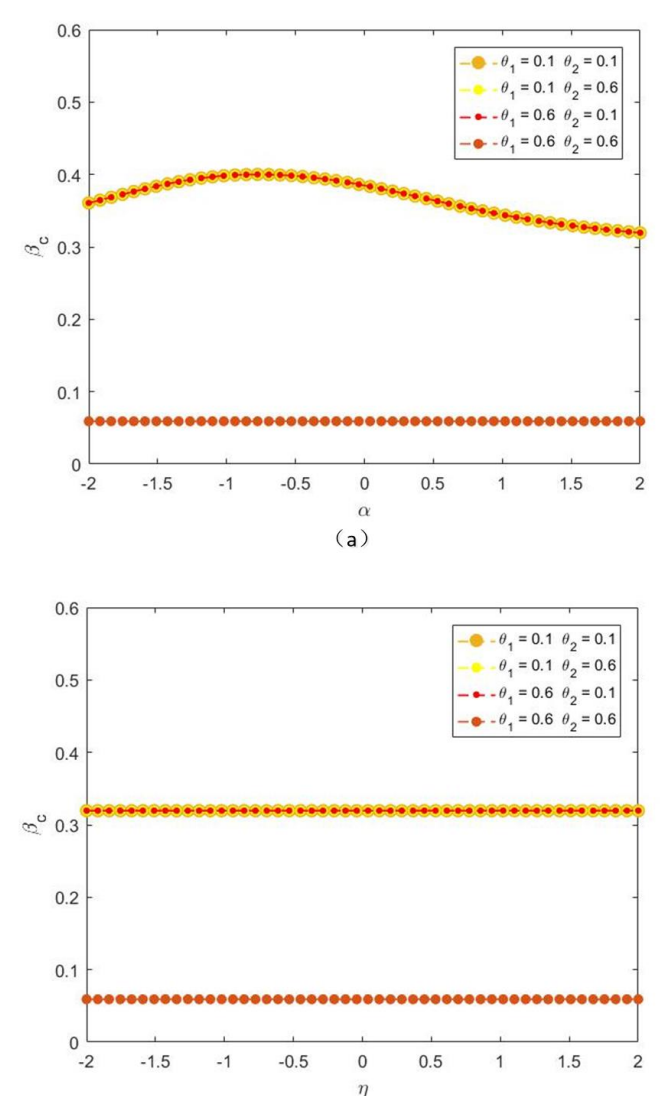

(b)

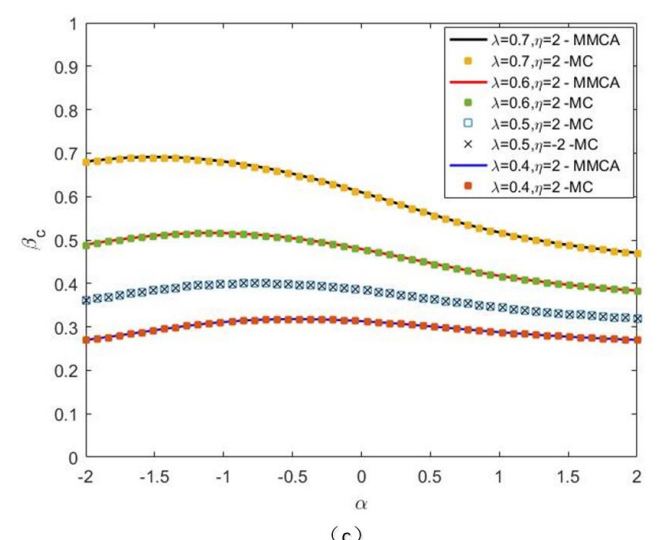

Fig. 8 (a) $\beta_{c}$ as a function of $\alpha$ for different values of $\theta_{1}$ and $\theta_{2}$, where $\lambda=0.5, \eta=2$. (b) $\beta_{c}$ as a function of $\eta$ for different values of $\theta_{1}$ and $\theta_{2}$, where $\lambda=0.5, \alpha=2$. (c) $\beta_{c}$ as a function of $\alpha$ for different values of $\lambda$ and $\eta$, where $\theta_{1}=0.1, \theta_{2}=0.1$. The three diagrams are obtained by averaging $50 \mathrm{MC}$ simulations. The remaining parameter are $\mu=0.8$, $\delta=0.6, \tau=0.2, \pi=0.5$.

that $\beta_{c}$ will undergo a second-order phase transition if $\theta_{2}$ (or $\theta_{1}$ ) increases alone. This is similar with the results of previous studies [27]. Moreover, when $\theta_{2}$ is around 0.73 , as $\theta_{1}$ raises, $\beta_{c}$ decreases first, then increases, and then decreases.

Then, we explore the impacts of the heterogeneity of individual influences in the information layer and the hetero- 

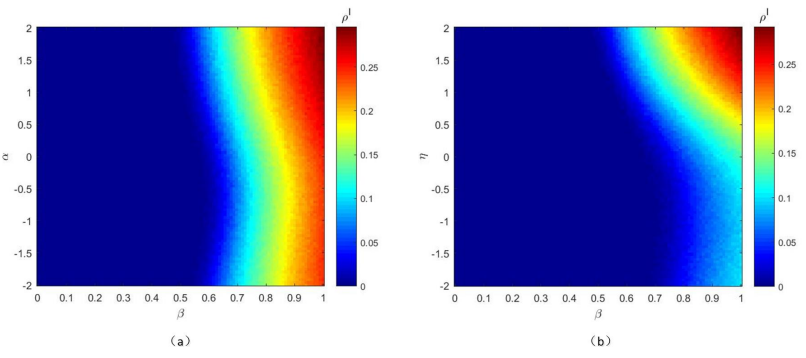

Fig. 9 (a) The full phase diagrams $(\alpha-\beta)$ of $\rho^{I}$, where $\eta=2$. (b) The full phase diagrams $(\eta-\beta)$ of $\rho^{I}$, where $\alpha=2$. The two phase diagrams are obtained by averaging $50 \mathrm{MC}$ simulations for each point in the grid $100 \times 100$. The remaining parameter are $\mu=0.8, \delta=0.6, \theta_{1}=0.1$, $\theta_{2}=0.1, \tau=0.2, \pi=0.5, \lambda=0.5$.

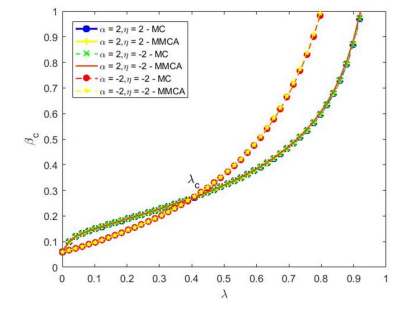

(a)

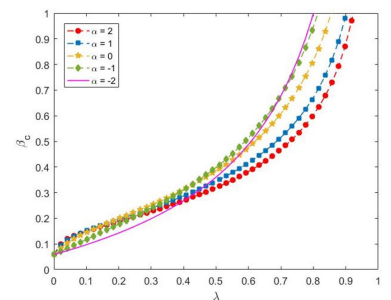

(b)
Fig. 10 (a) $\beta_{c}$ as a function of $\lambda$ for different values of $\alpha$ and $\eta$, where $\lambda_{c}$ represents the value of $\lambda$ when $\beta_{c}$ as the functional curves of $\lambda$ intersect for different values of $\alpha$. (b) $\beta_{c}$ as a function of $\lambda$ for different values of $\alpha$, where $\eta=-2$. The two diagrams are obtained by averaging $100 \mathrm{MC}$ simulations. The remaining parameter are $\mu=0.8, \delta=0.6, \theta_{1}=0.1$, $\theta_{2}=0.1, \tau=0.2, \pi=0.5$.

geneity of individual influences in the epidemic layer on epidemic transmission. In Fig.8, the results of MMCA and MC simulations are consistent. At the same time, the result show that $\theta_{1}$ or $\theta_{2}$ needs to be less than the threshold which makes $\beta_{c}$ undergo an abrupt transition at a certain $\theta_{1}$ or $\theta_{2}$, when $\alpha$ takes effect on $\beta_{c}$. Nevertheless, we find that the parameter $\eta$ has few effect on $\beta_{c}$ in these conditions. In addition, the parameter $\alpha$ has a nonlinear influence on $\beta_{c}$. For simplicity, we only consider that most of individuals are aware in Fig.8 (c). Thus, we set $\theta_{1}=0.1, \theta_{2}=0.1$. Note that $\beta_{c}$ increases as $\lambda$ raises. Hence, improving the strength of an individuals' responses to epidemics and moderately adjusting the heterogeneity of individual influences in the information layer can enhance the epidemic threshold.

In order to investigate the impacts of $\alpha$ and $\eta$ on the final density of infected individuals $\rho^{I}$, we plot the two phase diagrams as the shown in Fig.9. Compared Fig.8 with Fig.9, the variation of $\rho^{I}$ is related to the value of $\beta_{c}$ for $\alpha$, i.e., $\rho^{I}$ will increase as $\beta$ increases if $\beta$ is larger than $\beta_{c}$ for a certain $\alpha$. Meanwhile, $\rho^{I}$ increases as the parameter $\eta$ enhances, i.e., $\eta$ can affects the final density of infected individuals $\rho^{I}$. These results demonstrate that the parameter $\alpha$ has influences on the size of $\beta_{c}$ and $\rho^{I}$. However, the parameter $\eta$ has few effect on $\beta_{c}$, but can affects the magnitude of $\rho^{I}$.
In Fig.10, we find that the parameter $\lambda$ has a nonlinear effect on the epidemic threshold $\beta_{c}$. And then, $\beta_{c}$ enhances as the parameter $\lambda$ increases. Moreover, We assume that the value of $\lambda$ is $\lambda_{c}$ when $\beta_{c}$ as the functional curves of $\lambda$ intersect for different values of $\alpha$. When $\lambda<\lambda_{c}$, the greater $\alpha$, the larger $\beta_{c}$; When $\lambda>\lambda_{c}$, the smaller $\alpha$, the larger $\beta_{c}$. The results demonstrate that the epidemic threshold can be increased by adjusting the value of $\alpha$ when the power to improve the strength of an individuals' responses to epidemics are limited.

\section{Conclusion}

In this paper, to explore the interplay of awareness and epidemic, we propose a coupled awareness-epidemic spreading model considering the heterogeneity of individual influences. Being different from researches in the past, we take into account that the individuals' receptive preference for information and the impacts of the perceived local awareness ratio of individuals and individuals' perceived epidemic severity on self-protective behavior. Moreover, the processes of local and global awareness diffusion are described by the threshold model. The results show that the more individuals can accept information shared by others, the more beneficial to suppress the epidemic transmission. In addition, we find when $\theta_{2}$ (or $\theta_{1}$ ) increases alone, $\beta_{c}$ will undergo a secondorder phase transition, which is similar with the results of previous studies [27]. In our proposed model, the heterogeneity of individual influences in the epidemic layer has few effect on the epidemic threshold, but can affects the magnitude of the final density of infected individuals. Furthermore, we find that either the heterogeneity of individual influences in the information layer or the strength of individuals' responses has a nonlinear effect on the size of the epidemic threshold. At the same time, when the power to improve the strength of an individuals' responses to epidemics are limited, the epidemic threshold can be increased by adjusting the value of $\alpha$. In summary, our proposed model contributes to our understanding of the interaction between epidemic transmission and awareness diffusion, and provides some assistance on how to control the outbreak of an epidemic.

\section{References}

1. Ye, Y., Zhang, Q.P., Ruan, Z.Y., Cao, Z.D., Xuan, Q., Zeng, D.D.J.: Effect of heterogeneous risk perception on information diffusion, behavior change, and disease transmission. Phys. Rev. E 102, 042314 (2020)

2. Huang, H., Chen, Y.H., Yan, Z.J.: Impacts of social distancing on the spread of infectious diseases with asymptomatic infection: A mathematical model. Appl. Math. Comput. 398, 125983 (2021)

3. Yin, S., Zhang, N.: Prevention schemes for future pandemic cases: mathematical model and experience of interurban multi-agent COVID-19 epidemic prevention. Nonlinear Dyn 104, 2865-2900 (2021) 
4. Wang, X., Wang, S., Lan, Y., Tao, X., Xiao, J.: The impact of asymptomatic individuals on the strength of public health interventions to prevent the second outbreak of COVID-19. Nonlinear Dyn 101, 2003-2012 (2020)

5. Kabir, K.M.A., Tanimoto, J.: Analysis of epidemic outbreaks in two-layer networks with different structures for information spreading and disease diffusion. Commun Nonlinear Sci Numer Simul 72, 565-574 (2019)

6. Xian, J.J., Yang, D., Pan, L.M., Liu, M., Wang, W.: Containing rumors spreading on correlated multiplex networks. J. Stat. Mech. Theory Exp. 2020, 023402 (2020)

7. Wang, Z.S., Xia, C.Y., Chen,Z.Q., Chen,G.R.: Epidemic Propagation With Positive and Negative Preventive Information in Multiplex Networks. IEEE Trans Cybern 51, 1454-1462 (2021)

8. Huang, H., Chen, Y.H., Ma, Y.F.: Modeling the competitive diffusions of rumor and knowledge and the impacts on epidemic spreading. Appl. Math. Comput. 388, 125536 (2021)

9. Pan, Y.H., Yan, Z.J.: The impact of multiple information on coupled awareness-epidemic dynamics in multiplex networks. Physica A 491, 45-54 (2018)

10. Fan, C.J., Jin, Y., Huo, L.A., Liu, C., Yang, Y.P., Wang, Y.Q.: Effect of individual behavior on the interplay between awareness and disease spreading in multiplex networks. Physica A 461, 523-530 (2016)

11. Wang, H., Ma, C., Chen, H.S., Zhang, H.F.: Effects of asymptomatic infection and self-initiated awareness on the coupled disease-awareness dynamics in multiplex networks. Appl. Math. Comput. 400, 126084 (2021)

12. Hosni, A.I.E., Li, K., Ahmad, S.: Minimizing rumor influence in multiplex online social networks based on human individual and social behaviors. Inf Sci 512, 1458-1480 (2020)

13. Liu, Q.H., Wang, W., Tang, M., Zhang, H.F.: Impacts of complex behavioral responses on asymmetric interacting spreading dynamics in multiplex networks. Sci. Rep. 6, 25617 (2016)

14. Granell, C., Gomez, S., and Arenas, A.: Competing spreading processes on multiplex networks: awareness and epidemics. Phys. Rev. E 90, 012808 (2014)

15. Kan, J.Q., Ma, C., Zhang, H.F., Xiang, B.B.: Interplay of epidemic spreading and strategy-mixed awareness diffusion on multiplex networks. Int J Mod Phys C 31, 2050085 (2020)

16. Wu, Q.C., Fu, X.C., Small, M., Xu, X.J.: The impact of awareness on epidemic spreading in networks. Chaos 22, 013101 (2012)

17. Li, C., Zhang, Y., Li, X.: Epidemic Threshold in Temporal Multiplex Networks With Individual Layer Preference. IEEE Trans. Netw. Sci. Eng. 8, 814-824 (2021)

18. Funk, S., Gilad, E., Watkins, C., Jansen, V.A.A.: The spread of awareness and its impact on epidemic outbreaks. Proc. Natl. Acad. Sci. U.S.A. 106, 6872-6877 (2009)

19. Wang, W., Tang, M., Yang, H., Do, Y., Lai, Y.C., Lee, G.: Asymmetrically interacting spreading dynamics on complex layered networks. Sci. Rep. 4, 5097 (2014)

20. Wang, Z.S., Guo, Q.T., Sun, S.W., Xia, C.Y.: The impact of awareness diffusion on SIR-like epidemics in multiplex networks. Appl. Math. Comput. 349, 134-147 (2019)

21. Zhang, H.F., Xie, J.R., Tang, M., Lai, Y.C.: Suppression of epidemic spreading in complex networks by local information based behavioral responses. Chaos 24, 043106 (2014)

22. Guo, Q.T., Jiang, X., Lei, Y.J., Li, M., Ma, Y.F., Zheng, Z.M.: Twostage effects of awareness cascade on epidemic spreading in multiplex networks. Phys. Rev. E 91, 012822 (2015)

23. Kan, J.Q., Zhang, H.F.: Effects of awareness diffusion and selfinitiated awareness behavior on epidemic spreading - An approach based on multiplex networks. Commun Nonlinear Sci Numer Simul 44, 193-203 (2017)
24. Zuo, C., Wang, A.J., Zhu, F.P., Meng, Z.Y., Zhao, X.K.: A New Coupled Awareness-Epidemic Spreading Model with Neighbor Behavior on Multiplex Networks. Complexity 2021, 6680135 (2021)

25. Wang, Z.S., Xia, C.Y.: Co-evolution spreading of multiple information and epidemics on two-layered networks under the influence of mass media. Nonlinear Dyn. 102, 3039-3052 (2020)

26. Zhang, Y.X., Lu, X., Cui, N., Tang, J.T., Zhang, X.Y.: Coevolving Dynamics between Epidemic and Information Spreading considering the Dependence between Vigilance and Awareness Prevalence. Complexity 2021, 5515549 (2021)

27. Pan, Y.H., Yan, Z.J.: The impact of individual heterogeneity on the coupled awareness-epidemic dynamics in multiplex networks. Chaos 28, 063123 (2018)

28. Scatà, M., Di Stefano, A., Liò, P., La Corte, A.: The Impact of Heterogeneity and Awareness in Modeling Epidemic Spreading on Multiplex Networks. Sci. Rep. 6, 37105 (2016)

29. Chen, X.L., Gong, K., Wang, R.J., Cai, S.M., Wang, W.: Effects of heterogeneous self-protection awareness on resource-epidemic coevolution dynamics. Appl. Math. Comput. 385, 125428 (2020)

30. Barabási, A.L., Albert, R.: Emergence of Scaling in Random Networks. Science 286, 509-512 (1999)

31. Granell, C., Gómez, S., Arenas, A.: Dynamical Interplay between Awareness and Epidemic Spreading in Multiplex Networks. Phys. Rev. Lett. 111, 128701 (2013)

32. Chakrabarti, D., Wang, Y., Wang, C.X., Leskovec, J., Faloutsos, C.: Epidemic thresholds in real networks. ACM T INFORM SYST SE 10, 13 (2008)

\section{Statements and Declarations}

Funding This work was supported by the National Natural Science Foundation of China (61803184), The Youth Fund for Humanities and Social Sciences Research of The Ministry of Education (20YJC630059), National Nature Science Foundation of Jiangsu Province (BK20190832), China Postdoctoral Science Foundation (2018M640326, 2019M661731).

Competing Interests The authors have no relevant financial or non-financial interests to disclose.

Author Contributions All authors contributed to the study conception and design. Material preparation, data collection and analysis were performed by Haidong $\mathrm{Xu}$, Ye Zhao and Dun Han. The first draft of the manuscript was written by Haidong Xu and all authors commented on previous versions of the manuscript. All authors read and approved the final manuscript.

Availability of data and material All data and materials can be obtained under the proposed model and algorithms.

Conflict of interest The authors declare that they have no conflict of interest. 